\title{
Risk factors for unplanned readmission within 30 days after pediatric neurosurgery: a nationwide analysis of 9799 procedures from the American College of Surgeons National Surgical Quality Improvement Program
}

\author{
Brandon A. Sherrod, BS, James M. Johnston, MD, and Brandon G. Rocque, MD, MS \\ Department of Neurological Surgery, The University of Alabama at Birmingham, Alabama
}

OBJECTIVE Hospital readmission rate is increasingly used as a quality outcome measure after surgery. The purpose of this study was to establish, using a national database, the baseline readmission rates and risk factors for patient readmission after pediatric neurosurgical procedures.

METHODS The American College of Surgeons National Surgical Quality Improvement Program-Pediatric database was queried for pediatric patients treated by a neurosurgeon between 2012 and 2013. Procedures were categorized by current procedural terminology (CPT) code. Patient demographics, comorbidities, preoperative laboratory values, operative variables, and postoperative complications were analyzed via univariate and multivariate techniques to find associations with unplanned readmissions within 30 days of the primary procedure.

RESULTS A total of 9799 cases met the inclusion criteria, 1098 (11.2\%) of which had an unplanned readmission within 30 days. Readmission occurred $14.0 \pm 7.7$ days postoperatively (mean \pm standard deviation). The 4 procedures with the highest unplanned readmission rates were CSF shunt revision (17.3\%; CPT codes 62225 and 62230 ), repair of myelomeningocele $>5 \mathrm{~cm}$ in diameter (15.4\%), CSF shunt creation (14.1\%), and craniectomy for infratentorial tumor excision (13.9\%). The lowest unplanned readmission rates were for spine (6.5\%), craniotomy for craniosynostosis $(2.1 \%)$, and skin lesion (1.0\%) procedures. On multivariate regression analysis, the odds of readmission were greatest in patients experiencing postoperative surgical site infection (SSI; deep, organ/space, superficial SSI, and wound disruption: OR > 12 and $p<0.001$ for each). Postoperative pneumonia (OR 4.294, $p<0.001$ ), urinary tract infection (OR 4.262, $p<0.001$ ), and sepsis (OR 2.616, $p=0.006)$ also independently increased the readmission risk. Independent patient risk factors for unplanned readmission included Native American race (OR 2.363, $p=0.019)$, steroid use $>10$ days (OR 1.411, $p=$ 0.010 ), oxygen supplementation (OR 1.645, $p=0.010$ ), nutritional support (OR 1.403, $p=0.009$ ), seizure disorder (OR $1.250, p=0.021$ ), and longer operative time (per hour increase, $O R 1.059, p=0.029$ ).

CONCLUSIONS This study may aid in identifying patients at risk for unplanned readmission following pediatric neurosurgery, potentially helping to focus efforts at lowering readmission rates, minimizing patient risk, and lowering costs for health care systems.

http://thejns.org/doi/abs/10.3171/2016.2.PEDS15604

KEY WORDS National Surgical Quality Improvement Program; quality outcome; pediatric neurosurgery; readmission

$\mathrm{U}$ NPLANNED hospital readmissions after surgery present medical and financial challenges for health care systems and have emerged as an important measure of health care quality and efficiency., ${ }^{2,14,18}$ Recent health care reforms have led the Centers for Medicare \&
Medicaid Services to penalize providers for higher rates of unplanned readmissions. ${ }^{7}$ Furthermore, unplanned readmissions provide a quality outcome metric that may prove useful in quality improvement, patient risk stratification, and counseling patients and families prior to opera-

ABBREVIATIONS ACS = American College of Surgeons; ASA = American Society of Anesthesiologists; AST = aspartate transaminase; BUN = blood urea nitrogen; CPT = current procedural terminology; CVA = cerebrovascular accident; DVT = deep venous thrombosis; HIPAA = Health Insurance Portability and Accountability Act; ICD-9 = International Classification of Diseases, Ninth Revision; MMC = myelomeningocele; NSQIP-P = National Quality Improvement Program-Pediatric; PE = pulmonary embolism; PT = prothrombin time; $\mathrm{PTT}=$ partial thromboplastin time; $\mathrm{ROC}=$ receiver operating characteristic; $\mathrm{SIRS}=$ systemic inflammatory response syndrome; SSI = surgical site infection; UTI = urinary tract infection; WBC = white blood cell.

SUBMITTED October 18, 2015. ACCEPTED February 4, 2016.

INCLUDE WHEN CITING Published online May 17, 2016; DOI: 10.3171/2016.2.PEDS15604. 
tions..$^{2,14,18}$ Previous studies have demonstrated that readmission in pediatric patients can be accurately predicted from preexisting patient conditions and the severity of the condition necessitating admission. ${ }^{3,13}$

Unplanned readmission has not been well studied in pediatric neurosurgery despite growing attention paid to unplanned readmission as a quality outcome measure. Pediatric neurosurgery had the highest morbidity and mortality rates of any pediatric surgical specialty in a betaphase report of the American College of Surgeons (ACS) National Surgical Quality Improvement Program-Pediatric (NSQIP-P) database, indicating a need to assess patient risk factors for complications, readmission, and other outcome measures in neurosurgery. ${ }^{4}$ Previous studies have investigated return to the health care system (readmission or reoperation) after pediatric neurosurgery at a single institution ${ }^{9,21,26}$ and 30-day outcomes after pediatric shunt surgery; ${ }^{20}$ to our knowledge, however, no study has used a national, multiinstitutional patient database with followup to analyze risk factors for readmission after any pediatric neurosurgical procedure. Identifying the baseline rate of readmission for common pediatric neurosurgical procedures is useful to provide a benchmark for quality improvement efforts. Additionally, examining the risk factors for unplanned readmission will facilitate evidencebased patient risk stratification by health care systems to develop guidelines to reduce the likelihood of unplanned readmission.

The purpose of this study was to analyze patient and operative risk factors for unplanned readmission within 30 days of primary pediatric neurosurgical procedures by using a national surgical patient database with follow-up.

\section{Methods \\ Data Source}

The ACS-NSQIP-P is a nationwide, prospectively collected patient database with over 50 participating institutions and more than 300 patient variables. ${ }^{1}$ It includes the following de-identified and Health Insurance Portability and Accountability Act (HIPAA)-compliant variable categories: patient demographics, comorbidities, operative variables, preoperative lab values, primary procedure current procedural terminology (CPT) codes, International Classification of Diseases, Ninth Revision (ICD-9) codes, and 30-day postoperative events such as readmission, reoperation, mortality, and complications.

Rates of discrepancies between data abstractors are less than $2 \%$ as data abstractors are trained by the ACS to ensure the collection of high-quality data. ${ }^{1}$ Patient followup is performed by telephone or letter for a maximum of 30 days after discharge. ${ }^{1}$ Previous studies have shown that the NSQIP-P achieved 91.4\% confirmed 30-day followup. ${ }^{4}$ Institutional participation in the NSQIP is associated with reductions in postoperative adverse events ${ }^{11}$ and allows for a more thorough risk-adjusted analysis than an institutional administrative database. ${ }^{25}$ The NSQIP has been shown to capture all-cause and unplanned readmission occurrences more accurately than information from medical records regarding the cause of readmission. ${ }^{22}$ Our institution does not require institutional review board ap- proval for NSQIP studies given that NSQIP data are nonidentifiable and HIPAA compliant. ${ }^{24}$

\section{Data Acquisition}

We queried the NSQIP-P database for patients younger than 18 years (at the time of the procedure) who had undergone a procedure performed by a neurosurgeon or pediatric neurosurgeon during the period from 2012 to 2013. Procedures were grouped by CPT codes into the following procedural categories: spine; craniotomy for craniosynostosis; craniotomy for neoplasm; craniotomy for Chiari decompression; shunt or ventricular catheter placement; shunt or ventricular catheter revision, removal, or irrigation; myelomeningocele (MMC) repair; skin lesion; and other. Procedure categories and specific CPT codes within each category are listed in Fig. 1.

Patient demographic information included age, sex, and race. Patient comorbidities of interest included obesity, pulmonary comorbidity, gastrointestinal comorbidity, renal comorbidity, CNS comorbidity, cardiac comorbidity, steroid use (within 30 days before the principal procedure or at the time the patient was being considered as a candidate for surgery; it did not include short-term use, such as a 1-time pulse, limited short course, or taper of $\leq 10$ days), chemotherapy within 30 days prior to surgery, radiotherapy within 90 days prior to surgery, open wound (with or without infection), tracheostomy at the time of surgery, immune disease or immunosuppressant use, nutritional support (intravenous or nasogastric tube), bleeding disorder, hematological disorder, current or previous history of malignancy, history of prematurity, intraventricular hemorrhage, preoperative systemic inflammatory response syndrome (SIRS) or sepsis within 48 hours prior to surgery, and congenital malformation of any organ system (a detailed list of conditions included within each NSQIP-P categorical comorbidity variable is shown in Supplement S1).

Operative and hospital variables of interest included hospital length of stay; inpatient or outpatient status; concurrent procedure status; prior operation within 30 days; transfer status; discharge destination; operative time; American Society of Anesthesiologists (ASA) score; blood transfusion; elective, urgent, or emergent triage; and wound classification.

Preoperative lab values of interest included hypoalbuminemia, hyponatremia, hypernatremia, elevated white blood cell (WBC) count, thrombocytopenia, elevated aspartate transaminase (AST), elevated blood urea nitrogen (BUN), abnormal prothrombin time (PT), and abnormal partial thromboplastin time (PTT).

Noted postoperative complications included infections (surgical site infection [SSI], sepsis, urinary tract infection [UTI], pneumonia, central line bloodstream infection), wound disruption, unplanned intubation, renal failure or insufficiency, coma lasting more than 24 hours, cerebrovascular accident (CVA; stroke or intracerebral hemorrhage) or intracranial hemorrhage, seizure, peripheral nerve injury, cardiac arrest, graft or prosthesis failure, deep venous thrombosis (DVT), and pulmonary embolism (PE). The NSQIP-P data on time to postoperative complications were analyzed.

Unplanned readmission is defined in the NSQIP da- 


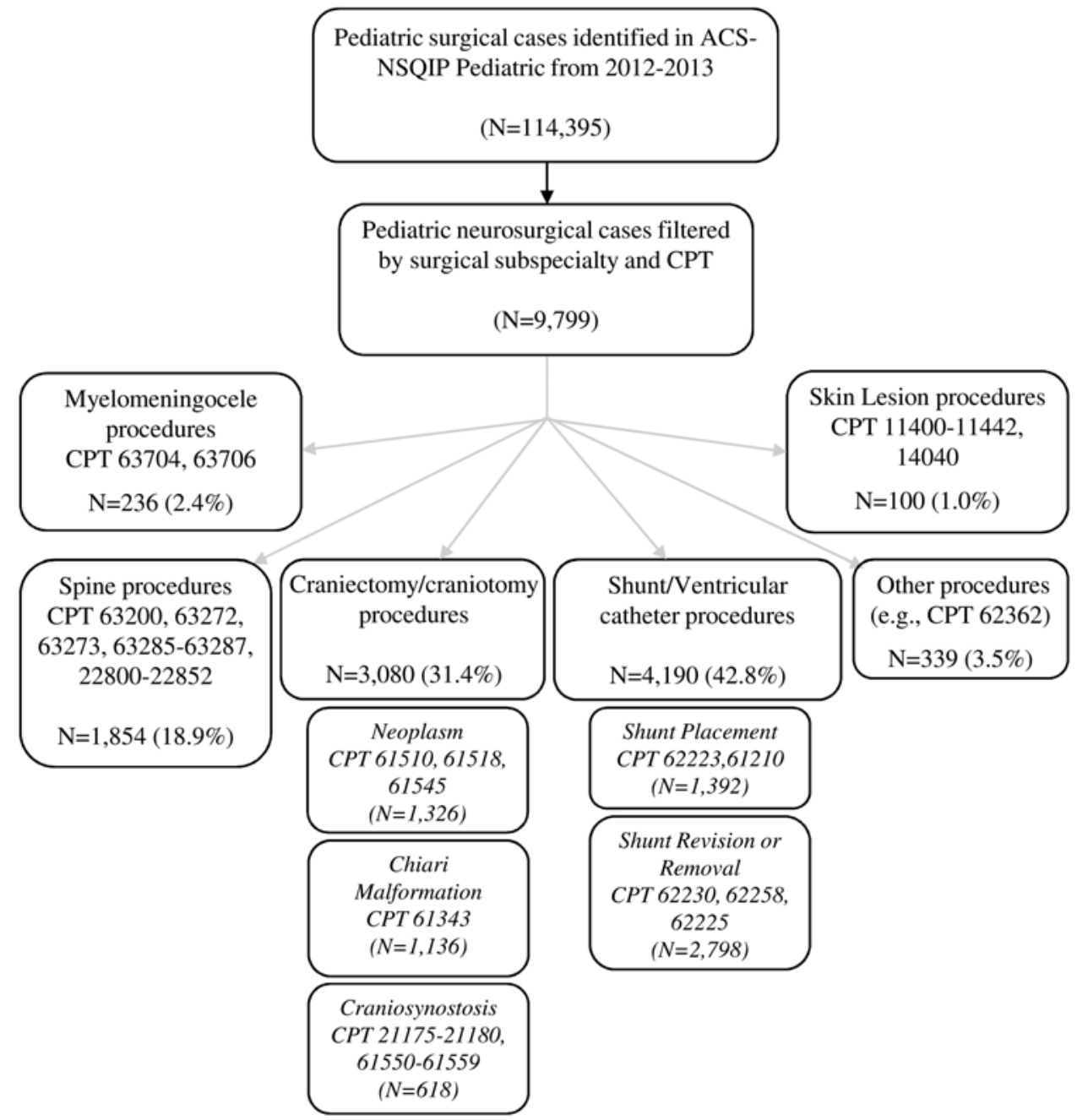

FIG. 1. Cohort selection by surgical subspecialty and procedural classification by CPT code. $N=$ number of cases.

tabase as "any unplanned readmission for any reason within 30 days of the principal surgical procedure. The readmission has to be classified as an 'inpatient' stay by the readmitting hospital, or reported by the patient/family as such."1 Unplanned readmission is coded in the NSQIP "if the readmission was unplanned." Previous studies have demonstrated that an unplanned readmission designation in the NSQIP has greater than 95\% agreement with hospital chart records regarding planned versus unplanned readmission designation. ${ }^{22}$

We defined readmission risk factors as any patient characteristic or event (pre- or postoperatively) that increased the likelihood of postoperative readmission. This definition included postoperative complications (for example, SSI) that could also be reasons for readmission.

\section{Data Analysis}

Univariate analysis of unplanned readmission outcome association with procedure type, patient demographics, patient comorbidities, preoperative lab values, operative variables, and postoperative complications was performed using the chi-square test, Fisher's exact test, or univariate logistic regression where appropriate. Variables with significance of $\mathrm{p} \leq 0.20$ in the univariate analyses were then entered into a multivariate analysis via binary logistic regression and were considered independently significant when $\mathrm{p} \leq 0.05$. As a secondary analysis, we applied a Bonferroni correction to the univariate analysis to correct for the higher risk of Type I error due to the use of over 100 independent variables. In this case, only variables with $\mathrm{p} \leq$ 0.002 in the univariate analysis were entered into the multivariate analysis (the $\alpha_{\text {corrected }}$ value for reaching significance in the Bonferroni correction is calculated by dividing the original $\alpha$ value - in our case, 0.2 - by the number of independent variables in the analysis). Receiver operating characteristic (ROC) curve analysis was performed for multivariate regression model validation. Statistical analyses were performed using SPSS version 22.0 (IBM Corp.).

\section{Results}

A total of 9799 cases met our inclusion criteria, 1098 (11.2\%) of which had an unplanned readmission within 30 days. An additional 166 readmissions were planned, making a total of 1264 postoperative readmissions within 30 days. The average time to unplanned readmission after the primary procedure was $14.04 \pm 7.74$ days (mean \pm SD). 
TABLE 1. Highest 30-day unplanned readmission rates by individual procedure

\begin{tabular}{clrrcc}
\hline CPT Code & \multicolumn{1}{c}{ Procedure Description } & No. & \% of All Procedures & \% Unplanned Readmissions \\
\hline 62225 & Replacement or irrigation of ventricular catheter & 867 & 8.8 & 19.7 \\
\hline 62230 & Replacement or revision of CSF shunt & 1521 & 15.5 & 16.0 \\
\hline 63706 & Repair of MMC 5-cm diameter & 123 & 1.3 & 15.4 \\
\hline 62223 & CSF shunt creation & 1072 & 10.9 & 14.1 \\
\hline 61518 & Craniectomy for infratentorial tumor excision & 561 & 5.7 & 13.9 \\
\hline
\end{tabular}

Procedures with the highest unplanned readmission rates included replacement or irrigation of ventricular catheter (CPT code 62225, commonly used to describe revision of the proximal catheter of a shunt system, 19.7\% readmitted), replacement or revision of CSF shunt (CPT code 62230, used to describe revision of a valve or distal catheter in a shunt system, 16.0\% readmitted), MMC repair > 5-cm diameter (CPT code 63706, 15.4\% readmitted), creation of CSF shunt (CPT code 62223, 14.1\% readmitted), and craniectomy for excision of infratentorial brain tumor (CPT code 61518, 13.9\% readmitted). Mortality for 3 of these procedures (MMC repair, shunt placement, and infratentorial tumor excision) was greater than the mortality for the general pediatric neurosurgical population in this study (among the 9799 cases). All but 1 of the 5 procedures with the highest readmission rates were relatively common, with each of the 4 accounting for more than $5 \%$ of the total procedures performed (MMC repair $>5 \mathrm{~cm}$ accounted for only $1.3 \%$ of all procedures). The highest rates of unplanned readmission by procedure are shown in Table 1.

Rates of unplanned readmission varied significantly according to procedural category (as defined by lists in Fig. 1), ranging from $1.0 \%$ for skin lesion procedures to $16.8 \%$ for shunt revision, removal, and irrigation procedures. Two procedure categories showed no significant difference in the rate of unplanned readmission compared with all other procedures: craniotomy for neoplasm and other (primarily baclofen pump placement procedures). Procedure category associations with unplanned readmission can be found in Table 2 .
Some patient characteristics and demographics, including length of hospitalization, prior operation within 30 days of the current procedure, admission through the emergency room, discharge to home compared to other types of discharges, and Native American race, were significantly different between readmitted and nonreadmitted groups on univariate analysis. No difference was seen for age, neonate status, patient sex, inpatient or outpatient status, white race, African American race, Asian race, $\mathrm{Pa}$ cific Islander race, unknown race, concurrent procedure, or transfer from an outside hospital or rehab facility. A longer hospitalization was a significant protective factor for unplanned readmission via univariate logistic regression $(\mathrm{OR}=0.993$ per day increase, $95 \% \mathrm{CI}=0.987-0.999$, $\mathrm{p}=0.033)$. Patient demographics and characteristics are displayed in Table 3.

Several patient comorbidities were significantly associated with unplanned readmission on univariate analysis, including the presence of any comorbidity, the presence of any non-CNS comorbidity, pulmonary comorbidity (specific conditions significantly associated with readmission: asthma, bronchopulmonary dysplasia, oxygen supplementation, and structural pulmonary abnormality), gastrointestinal comorbidity (specific conditions significantly associated with readmission: esophageal, gastric, or intestinal disease), CNS comorbidity (specific conditions significantly associated with readmission: history of CVA or traumatic brain injury, CNS tumor, developmental delay, cerebral palsy, seizure disorder, and structural CNS abnormality), steroid use $>10$ days, chemotherapy within 30 days prior to surgery, radiotherapy within 90 days prior

TABLE 2. Procedure category association with unplanned readmission on univariate analysis*

\begin{tabular}{|c|c|c|c|c|c|c|}
\hline \multirow[b]{2}{*}{ Procedure Category } & \multirow[b]{2}{*}{$\%$ Readmitted } & \multicolumn{2}{|c|}{ No. $(\%)$} & \multirow[b]{2}{*}{ Crude OR† } & \multirow[b]{2}{*}{$95 \% \mathrm{Cl}$} & \multirow[b]{2}{*}{ p Value } \\
\hline & & $\begin{array}{c}\text { Unplanned } \\
\text { Readmission }\end{array}$ & $\begin{array}{l}\text { No Unplanned } \\
\text { Readmission }\end{array}$ & & & \\
\hline Shunt/ventricular catheter revision, removal, irrigation & 16.8 & $471(42.9)$ & $2327(26.7)$ & 2.058 & $1.809-2.340$ & $<0.001$ \\
\hline MMC repair & 14.4 & $34(3.1)$ & $202(2.3)$ & 1.344 & $0.930-1.944$ & 0.117 \\
\hline Shunt/ventricular catheter placement & 13.4 & $187(17.0)$ & $1205(13.8)$ & 1.277 & $1.079-1.512$ & 0.005 \\
\hline Craniotomy for neoplasm & 11.8 & $157(14.3)$ & $1169(13.4)$ & 1.076 & $0.899-1.288$ & 0.426 \\
\hline Other & 10.6 & $36(3.3)$ & $303(3.5)$ & 0.940 & $0.661-1.335$ & 0.793 \\
\hline Craniotomy for Chiari malformation & 7.0 & $79(7.2)$ & $1057(12.1)$ & 0.561 & $0.442-0.711$ & $<0.001$ \\
\hline Spine & 6.5 & $120(10.9)$ & $1734(19.9)$ & 0.493 & $0.405-0.600$ & $<0.001$ \\
\hline Craniotomy for craniosynostosis & 2.1 & $13(1.2)$ & $605(7.0)$ & 0.160 & $0.092-0.279$ & $<0.001$ \\
\hline Skin lesion & 1.0 & $1(0.1)$ & $99(1.1)$ & 0.079 & $0.011-0.568$ & $<0.001$ \\
\hline
\end{tabular}

* Statistical analysis was performed using binary logistic regression and chi-square test. Complete procedure categorization, including CPT codes within each category, appears in Fig. 1.

† Unadjusted odds ratio with respect to each variable's null state; that is, the odds ratio is in reference to all other procedures not in that individual category.

$\ddagger$ Primarily baclofen pump placement ( $82 \%$ of procedures in this category). 
TABLE 3. Patient demographic association with unplanned readmission on univariate analysis*

\begin{tabular}{|c|c|c|c|c|c|}
\hline Parameter & $\begin{array}{l}\text { Unplanned } \\
\text { Readmission }\end{array}$ & $\begin{array}{l}\text { No Unplanned } \\
\text { Readmission }\end{array}$ & Crude OR† & $95 \% \mathrm{Cl}$ & $\mathrm{p}$ Value \\
\hline Age in yrs (range) & $5.3(1.1-12.0)$ & $5.8(1.0-11.9)$ & 1.002 & $0.991-1.013$ & 0.781 \\
\hline No. of neonates (\%) & $79(7.2)$ & $649(7.4)$ & 0.962 & $0.755-1.226$ & 0.807 \\
\hline Total LOS in days (range) & $3(2-7)$ & $3(2-5)$ & 0.993 & $0.987-0.999$ & 0.033 \\
\hline \multicolumn{6}{|l|}{$\operatorname{Sex}($ no. $[\%])$} \\
\hline Male & $593(54.0)$ & $4661(53.6)$ & 1.018 & $0.897-1.154$ & 0.797 \\
\hline Female & $505(46.0)$ & $4040(46.4)$ & Ref & & \\
\hline Reported race (no. [\%]) & & & & & 0.183 \\
\hline White & $813(74.0)$ & $6391(73.4)$ & Ref & & \\
\hline African American & $149(13.6)$ & $1157(13.3)$ & 1.012 & $0.841-1.219$ & 0.897 \\
\hline Asian & $25(2.3)$ & $194(2.2)$ & 1.013 & $0.664-1.546$ & 0.952 \\
\hline Native American & $12(1.1)$ & $45(0.5)$ & 2.096 & $1.104-3.979$ & 0.024 \\
\hline Pacific Islander & $2(0.2)$ & $22(0.2)$ & 0.715 & $0.168-3.045$ & 0.650 \\
\hline Unknown & $97(8.8)$ & $892(10.2)$ & 0.855 & $0.685-1.067$ & 0.166 \\
\hline Patient status (no. [\%]) & & & & & 0.213 \\
\hline Inpatient & $1038(94.5)$ & $8135(93.5)$ & Ref & & \\
\hline Outpatient & $60(5.5)$ & $566(6.5)$ & 0.831 & $0.632-1.092$ & \\
\hline Prior operation w/in 30 days (no. [\%]) & $62(5.6)$ & $348(4.0)$ & 1.436 & $1.088-1.896$ & 0.013 \\
\hline Concurrent procedure (no. [\%]) & $54(4.9)$ & $475(5.4)$ & 0.896 & $0.671-1.196$ & 0.523 \\
\hline Transfer status (no. [\%]) & & & & & $<0.001$ \\
\hline Admitted from home, clinic, doctor's office & $620(56.5)$ & $6117(70.3)$ & Ref & & \\
\hline Admitted through ER & $390(35.5)$ & $1852(21.3)$ & 2.078 & $1.812-2.383$ & $<0.001$ \\
\hline Transfer from outside hospital or rehab facility & $88(8.0)$ & $732(8.4)$ & 1.186 & $0.937-1.502$ & 0.156 \\
\hline \multicolumn{6}{|l|}{ Discharge destination (no. [\%]) } \\
\hline Home & $1067(97.2)$ & $8294(95.3)$ & Ref & & \\
\hline Other (rehab, separate facility, skilled care, unskilled care) & $31(2.8)$ & 407 (4.7) & 0.592 & $0.409-0.858$ & 0.006 \\
\hline
\end{tabular}

to surgery, open wound, tracheostomy at time of surgery, history of prematurity (specifically, 25-28 weeks gestation), intraventricular hemorrhage, nutritional support, and current or previous malignancy. No difference was observed for obesity, renal comorbidity, cardiac comorbidity, immune disease or immunosuppressant use, bleeding disorder, hematological disorder, gestational period $>28$ weeks, SIRS or sepsis within 48 hours prior to surgery, or congenital malformation. Patient comorbidities are shown in Table 4.

No preoperative laboratory values were significantly associated with unplanned readmission on univariate analysis. Hypoalbuminemia and elevated WBC count alone met criteria for inclusion in the multivariate model $(\mathrm{p}<0.2)$. No difference was seen for hyponatremia, hypernatremia, thrombocytopenia, elevated AST, elevated BUN, abnormal PT, abnormal PTT, or anemia. Preoperative laboratory values are displayed in Table 5. Of note, not all patients had preoperative laboratory values measured.

Operative variables significantly associated with unplanned readmission on univariate analysis included a shorter operation, triage status, and ASA classification. No difference was observed for wound classification over- all ( $\mathrm{p}=0.310)$; however, dirty and/or infected wound status was entered into the multivariate analysis $(\mathrm{p}<0.2)$. Similarly, perioperative blood transfusion $(\mathrm{p}=0.129)$ was included in the multivariate analysis. Although a longer operation was protective for readmission on univariate logistic regression $(\mathrm{OR}=0.960$ per hour increase, $95 \% \mathrm{CI}$ $=0.925-0.995, \mathrm{p}=0.027)$, a longer operation emerged as an independently significant risk factor for readmission on multivariate analysis $(\mathrm{OR}=1.059$ per hour increase, $95 \%$ $\mathrm{CI}=1.006-1.114, \mathrm{p}=0.029)$. When all variables in the multivariate analysis were accounted for, the protective effect of a longer operation (a $4 \%$ decrease in readmission risk per hour increase in operative time) diminished and actually became a risk factor (a 5\% increase in readmission risk per hour increase in operative time). This was expected, as longer operations are typically performed in patients with more severe underlying conditions and are more likely to result in postoperative complications. Operative variable analysis is shown in Table 6.

Postoperative complications (systemic infections or SSIs, seizure, coma, unplanned reintubation, nerve injury, organ failure, graft failure, venous thromboembolism) occurred in 1237 cases $(12.6 \%)$ and were the strongest pre- 
TABLE 4. Patient comorbidities associated with unplanned readmission on univariate analysis*

\begin{tabular}{|c|c|c|c|c|c|}
\hline \multirow[b]{2}{*}{ Parameter } & \multicolumn{2}{|c|}{ No. $(\%)$} & \multirow[b]{2}{*}{ Crude OR† } & \multirow[b]{2}{*}{$95 \% \mathrm{Cl}$} & \multirow[b]{2}{*}{ p Value } \\
\hline & Unplanned Readmission & No Unplanned Readmission & & & \\
\hline Any comorbidity & 1069 (97.4) & $7877(90.5)$ & 3.856 & $2.648-5.165$ & $<0.001$ \\
\hline Any non-CNS comorbidity & $620(56.5)$ & $3821(43.9)$ & 1.657 & $1.460-1.880$ & $<0.001$ \\
\hline Obesity, BMI for age & $145(13.2)$ & $1148(13.2)$ & 1.001 & $0.832-1.205$ & 1.000 \\
\hline Pulmonary comorbidity & $227(20.7)$ & $1481(17.0)$ & 1.271 & $1.087-1.486$ & 0.003 \\
\hline Ventilator dependent & $37(3.4)$ & $325(3.7)$ & 0.899 & $0.636-1.270$ & 0.610 \\
\hline Pneumonia & $2(0.2)$ & $20(0.2)$ & 0.792 & $0.185-3.393$ & 1.000 \\
\hline Asthma & $78(7.1)$ & $501(5.8)$ & 1.252 & $0.977-1.603$ & 0.077 \\
\hline Cystic fibrosis & $1(0.1)$ & $5(0.0)$ & 1.585 & $0.185-13.583$ & 0.510 \\
\hline Bronchopulmonary dysplasia & $86(7.8)$ & $492(5.7)$ & 1.418 & $1.117-1.799$ & 0.005 \\
\hline Oxygen support & $72(6.6)$ & $421(4.8)$ & 1.380 & $1.066-1.787$ & 0.019 \\
\hline Structural pulmonary abnormality & $71(6.5)$ & $400(4.6)$ & 1.435 & $1.106-1.787$ & 0.009 \\
\hline GI comorbidity & $222(20.2)$ & $1257(14.4)$ & 1.501 & $1.280-1.759$ & $<0.001$ \\
\hline Esophageal, gastric, intestinal disease & $218(19.8)$ & $1228(14.1)$ & 1.508 & $1.285-1.769$ & $<0.001$ \\
\hline Biliary, liver, pancreatic disease & $9(0.8)$ & $54(0.6)$ & 1.323 & $0.652-2.688$ & 0.421 \\
\hline Renal comorbidity & $1(0.1)$ & $7(0.1)$ & 1.132 & $0.139-9.211$ & 1.000 \\
\hline Renal failure & $1(0.1)$ & $4(0.0)$ & 1.982 & $0.221-17.749$ & 0.448 \\
\hline Dialysis & $1(0.1)$ & $4(0.0)$ & 1.982 & $0.221-17.749$ & 0.448 \\
\hline CNS comorbidity & $1036(94.4)$ & $7606(87.4)$ & 2.406 & $1.848-3.132$ & $<0.001$ \\
\hline Coma $>24$ hrs & $0(0.0)$ & $5(0.0)$ & NA & NA & NA \\
\hline History of CVA or TBI & $95(8.6)$ & $534(6.1)$ & 1.449 & $1.153-1.819$ & 0.002 \\
\hline CNS tumor & $218(19.8)$ & $1360(15.6)$ & 1.337 & $1.140-1.568$ & $<0.001$ \\
\hline Developmental delay & $371(33.8)$ & $2273(26.1)$ & 1.443 & $1.262-1.650$ & $<0.001$ \\
\hline Cerebral palsy & $129(11.7)$ & $704(8.1)$ & 1.512 & $1.239-1.846$ & $<0.001$ \\
\hline Neuromuscular disorder & $112(10.2)$ & $840(9.6)$ & 1.063 & $0.863-1.309$ & 0.552 \\
\hline Seizure disorder & $254(23.1)$ & $1324(15.2)$ & 3.629 & $2.209-5.961$ & $<0.001$ \\
\hline Structural CNS abnormality & $862(78.5)$ & $6342(72.9)$ & 1.359 & $1.168-1.581$ & $<0.001$ \\
\hline Cardiac comorbidity & $126(11.5)$ & $922(10.6)$ & 1.094 & $0.897-1.333$ & 0.378 \\
\hline Steroid usef & $138(12.6)$ & $678(7.8)$ & 1.701 & $1.400-2.067$ & $<0.001$ \\
\hline Chemotherapy w/in 30 days before surgery & $33(3.0)$ & $109(1.2)$ & 2.442 & $1.646-3.642$ & $<0.001$ \\
\hline Radiotherapy w/in 90 days before surgery & $10(0.9)$ & $28(0.3)$ & 2.847 & $1.379-5.877$ & 0.008 \\
\hline Open wound (w/ or w/o infection) & $39(3.6)$ & $188(2.2)$ & 1.668 & $1.174-2.368$ & 0.007 \\
\hline Tracheostomy at time of surgery & $32(2.9)$ & $145(1.7)$ & 1.771 & $1.202-2.611$ & 0.005 \\
\hline Immune disease or immunosuppressant use & $13(1.2)$ & $74(0.8)$ & 1.397 & $0.772-2.527$ & 0.302 \\
\hline Nutritional support (IV or NG tube) & $168(15.3)$ & $833(9.6)$ & 1.706 & $1.426-2.041$ & $<0.001$ \\
\hline Bleeding disorder & $9(0.8)$ & $53(0.6)$ & 1.349 & $0.663-2.741$ & 0.416 \\
\hline Hematological disorder & $36(3.3)$ & $240(2.8)$ & 1.195 & $0.837-1.706$ & 0.332 \\
\hline Current or previous malignancy & $191(17.4)$ & $1046(12.0)$ & 1.541 & $1.302-1.825$ & $<0.001$ \\
\hline History of prematurity & & & & & 0.002 \\
\hline Term birth ( $\geq 37$ wks gestation) & $744(67.8)$ & $6167(70.9)$ & Ref & & \\
\hline 33-36 wks gestation & $97(8.8)$ & $732(8.4)$ & 1.098 & $0.877-1.376$ & 0.414 \\
\hline 29-32 wks gestation & $50(4.6)$ & $378(4.3)$ & 1.096 & $0.809-1.487$ & 0.554 \\
\hline 25-28 wks gestation & $110(10.0)$ & $593(6.8)$ & 1.538 & $1.237-1.911$ & $<0.001$ \\
\hline$\leq 24$ wks gestation & $36(3.3)$ & $241(2.8)$ & 1.238 & $0.865-1.772$ & 0.243 \\
\hline Unknown & $61(5.6)$ & $590(6.8)$ & 0.857 & $0.651-1.128$ & 0.270 \\
\hline Intraventricular hemorrhage & $141(12.8)$ & $873(10.0)$ & 1.321 & $1.092-1.598$ & 0.005 \\
\hline Congenital malformation, any system & $402(36.6)$ & $3247(37.3)$ & 0.970 & $0.852-1.105$ & 0.667 \\
\hline SIRS/sepsis w/in 48 hrs before surgery & $19(1.7)$ & $130(1.5)$ & 1.161 & $0.714-1.887$ & 0.514 \\
\hline
\end{tabular}




\section{» CONTINUED FROM PAGE 355}

\section{TABLE 4. Patient comorbidities associated with unplanned readmission on univariate analysis*}

$\mathrm{BMI}=$ body mass index; $\mathrm{GI}$ = gastrointestinal; IV = intravenous; $\mathrm{NA}=$ not applicable; NG = nasogastric; $\mathrm{TBI}$ = traumatic brain injury

* Statistical analysis was performed using Fisher's exact test, chi-square test, or logistic regression, as appropriate.

$\dagger$ Unadjusted odds ratio is in reference to the null state for variables without an explicitly defined reference category; that is, the odds ratio is in reference to cases without the comorbidity listed.

$\ddagger$ Steroid use is defined as the use of oral or parenteral corticosteroid within 30 days prior to the principal procedure or at the time the patient is being considered as a candidate for surgery; it does not include short-term use such as a 1-time pulse, limited short course, or a taper $<10$ days.

dictors of unplanned readmission. Data on days to postoperative complications are displayed in Fig. 2. Complications significantly associated with unplanned readmission on univariate analysis included any complication, any infection (superficial SSI, deep SSI, organ/space SSI, sepsis, UTI, pneumonia, central line-associated bloodstream infection), wound disruption, unplanned intubation, CVA, seizure, graft or prosthesis failure, and DVT. No differences were seen for renal insufficiency, acute renal failure, coma $>24$ hours, peripheral nerve injury, PE, or cardiac arrest (some events were not statistically testable due to a prohibitively low number of events). Postoperative complications analysis is displayed in Table 7.

For shunt operations with the highest readmission rates (CPT codes 62223, 62230, 62225; shown in Table 1), the overall 30-day unplanned readmission rate was $16.3 \%$. Within these 3 shunt operations, the most common reasons for readmission were shunt failure or mechanical device complication (ICD-9 code 996.2, 31.2\% of readmissions), need for another shunt procedure (CPT codes $62223-62258,17.9 \%$ of readmissions), and organ or space SSI (8.5\% of readmissions). Other less common reasons for readmission among the 3 shunt operations with the highest readmission rate included headache $(2.5 \%)$, wound disruption (2.1\%), and seizure (2.0\%). Similarly, in patients who underwent craniectomy for excision of brain infratentorial tumor (CPT code 61518, 30-day unplanned readmission rate of $13.9 \%$ ), the most common reasons for readmission included hydrocephalus (19.2\% of readmissions), wound disruption (11.5\% of readmissions), and organ or space SSI (7.7\% of readmissions).
Results from the multivariate logistic regression analysis are shown in Table 8. Numerous variables emerged as independently significant risk factors, including (in order of decreasing odds ratio): deep incisional SSI; organ or space SSI; wound disruption; superficial incisional SSI; graft or prosthesis failure; postoperative pneumonia; postoperative UTI; postoperative sepsis; postoperative seizure; Native American race; shunt or ventricular catheter removal, replacement, or irrigation procedure; shunt or ventricular catheter placement procedure; MMC repair procedure; presence of any comorbidity; home discharge; oxygen supplementation; steroid use $>10$ days; nutritional support; prior operation within 30 days of the current procedure; transfer from emergency room; preexisting seizure disorder; and longer operative time. Several factors emerged as independently significant protective factors for readmission, including longer hospital stay, spine procedure, and craniotomy for craniosynostosis procedure. Increasing length of stay proved to be a significant protective factor $(\mathrm{OR}=0.956$ per day increase, $95 \%$ CI $0.946-0.966$, $\mathrm{p}<0.001$ ); however, this variable is not included in Table 8 because of the decreasing time window in which readmission can occur as the length of stay increases. Variables that did not remain in the model when applying a Bonferroni correction for multiple measures were Native American race, oxygen supplementation, home discharge, prior operation within 30 days of index procedure, longer operative time, MMC repair procedures, and shunt placement procedures (marked with daggers in Table 8).

Receiver operating characteristic analysis for validation of the logistic regression model yielded a c-statistic, or area

TABLE 5. Preoperative laboratory value association with unplanned readmission on univariate analysis*

\begin{tabular}{lccrrr}
\hline & \multicolumn{3}{c}{ No. (\%) } & & \\
Parameter (\% w/ lab value) $\dagger$ & Unplanned Readmission & No Unplanned Readmission & Crude OR $\ddagger$ & 95\% Cl & $\mathrm{p} \mathrm{Value}$ \\
\hline Hypoalbuminemia (19.7) & $51(4.6)$ & $320(3.7)$ & 1.276 & $0.943-1.726$ & 0.130 \\
\hline Hyponatremia (58.0) & $42(3.8)$ & $280(3.2)$ & 1.196 & $0.859-1.665$ & 0.281 \\
\hline Hypernatremia (58.0) & $17(1.5)$ & $114(1.3)$ & 1.185 & $0.709-1.980$ & 0.486 \\
\hline Elevated WBC count (67.4) & $78(7.1)$ & $496(5.7)$ & 1.265 & $0.988-1.620$ & 0.066 \\
\hline Thrombocytopenia (67.0) & $57(5.2)$ & $398(4.6)$ & 1.142 & $0.859-1.519$ & 0.361 \\
\hline Elevated AST (18.1) & $14(1.3)$ & $115(1.3)$ & 0.964 & $0.552-1.685$ & 1.000 \\
\hline Elevated BUN (55.2) & $5(0.4)$ & $51(0.6)$ & 0.776 & $0.309-1.948$ & 0.831 \\
\hline Abnormal PT (36.4) & $53(4.8)$ & $370(4.2)$ & 1.142 & $0.850-1.534$ & 0.386 \\
\hline Abnormal PTT (37.5) & $65(5.9)$ & $516(5.9)$ & 0.998 & $0.765-1.302$ & 1.000 \\
\hline Anemia (68.2) & $89(8.1)$ & $618(7.1)$ & 1.154 & $0.915-1.454$ & 0.239 \\
\hline
\end{tabular}

* Statistical analysis was performed using logistic regression and Fisher's exact test.

$\dagger$ Not all patients had the listed preoperative lab measurements recorded; percentage refers to that of the total patient cohort with preoperative lab measurements, normal or abnormal.

$\ddagger$ Unadjusted odds ratio is in reference to the null state, that is, in relation to cases in which the given lab value is normal. 
TABLE 6. Operative variable association with unplanned readmission on univariate analysis*

\begin{tabular}{|c|c|c|c|c|c|}
\hline Parameter & Unplanned Readmission & No Unplanned Readmission & $\begin{array}{c}\text { Crude } \\
\text { OR† }\end{array}$ & $95 \% \mathrm{Cl}$ & $\mathrm{p}$ Value \\
\hline Length of operation in min (range) & $69(41-139)$ & $82(47-159)$ & 0.960 & $0.925-0.995$ & 0.027 \\
\hline Triage (no. [\%]) & & & & & $<0.001$ \\
\hline Elective & $685(62.4)$ & $6551(75.3)$ & Ref & & \\
\hline Emergent & $248(22.6)$ & $1198(13.8)$ & 1.980 & $1.691-2.318$ & $<0.001$ \\
\hline Urgent & $165(15.0)$ & $952(10.9)$ & 1.658 & $1.380-1.991$ & $<0.001$ \\
\hline ASA Class (no. [\%]) & & & & & $<0.001$ \\
\hline 1 & $20(1.8)$ & $476(5.5)$ & Ref & & \\
\hline II & $334(30.4)$ & $3438(39.5)$ & 2.312 & $1.458-3.667$ & $<0.001$ \\
\hline III & $692(63.0)$ & $4411(50.7)$ & 3.734 & $2.370-5.882$ & $<0.001$ \\
\hline IV & $48(4.4)$ & $346(4.0)$ & 3.302 & $1.925-5.664$ & $<0.001$ \\
\hline V & $0(0.0)$ & $12(0.1)$ & NA & NA & NA \\
\hline Unknown & $4(0.4)$ & $18(0.2)$ & 5.289 & $1.638-17.077$ & 0.005 \\
\hline Periop blood transfusion (no. [\%]) & $70(6.4)$ & $667(7.7)$ & 0.820 & $0.636-1.058$ & 0.129 \\
\hline Wound classification (no. [\%]) & & & & & 0.310 \\
\hline Clean & $1044(95.1)$ & $8362(96.1)$ & Ref & & \\
\hline Clean-contaminated & $30(2.7)$ & $206(2.4)$ & 1.166 & $0.791-1.720$ & 0.437 \\
\hline Contaminated & $9(0.8)$ & $57(0.7)$ & 1.265 & $0.624-2.562$ & 0.514 \\
\hline Dirty or infected & $15(1.4)$ & $76(0.9)$ & 1.581 & $0.905-2.761$ & 0.107 \\
\hline
\end{tabular}

* Statistical analysis was performed using Fisher's exact test, chi-square test, or univariate logistic regression, as appropriate. Continuous variables are expressed as the median (25th percentile-75th percentile).

† Unadjusted odds ratio; for categorical variables without an explicit reference category, odds ratios are in reference to cases in which the variable is not true (null state).

under the curve, of 0.759 (95\% CI $0.744-0.775, \mathrm{p}<0.001)$. An area under the curve $\geq 0.7$ by ROC analysis indicates an acceptable multivariate logistic regression model with significantly greater predictive ability than chance alone.

\section{Discussion}

We have identified rates of unplanned readmission as well as independent risk factors for readmission in a national pediatric neurosurgical population. To our knowledge, this is the first study in which the NSQIP database has been used to examine rates and risk factors for readmission after pediatric neurosurgery. While previous studies have reported return to system after pediatric neurosurgery at single institutions ${ }^{9,21,26}$ or after pediatric shunt surgery, ${ }^{20}$ the statistical power of a large national patient sample may provide a more representative picture of readmission outcomes for general pediatric neurosurgical procedures.

\section{Complications}

Postoperative infection, particularly SSI, was the strongest predictor of readmission. This observation is not surprising given that SSI is not uncommon and is associated with significant morbidity and mortality, particularly if not caught and treated early. Surgical site infection has been shown to cause up to $70 \%$ of reoperations after pediatric spine surgery ${ }^{17}$ and is one of the strongest predictors of readmission after pediatric plastic surgery. ${ }^{23}$ However, the relatively high rate of SSI and readmissions related to SSI could indicate a need to continue to study and improve preventative measures before, during, and after surgery (prophylactic antibiotic use, close monitoring of sterile technique, postoperative wound monitoring or cleaning) to prevent occurrences of SSI and SSI-related return to system. Additionally, wound disruption was a common reason for readmission and was independently associated with readmission.

Shunt failure was the most common ICD-9 diagnosis on readmission. In pediatric neurosurgery, shunt failure is a common and oftentimes unpreventable phenomenon, occurring in approximately $12 \%$ of pediatric shunt procedures within 30 days or less, ${ }^{11}$ and up to $46 \%$ of procedures had reported failure rates when the follow-up period lasted up to 1 year. ${ }^{6,10}$ The 30-day readmission rate for shunt operations (15.7\%) in this study is substantially similar to that published in previous work ${ }^{20}$ and may be useful as a benchmark for future studies aimed at lowering adverse shunt surgery events. As expected, the majority of readmissions after a CSF shunting procedure were related to recurrent shunt difficulties. Aside from shunt failure, however, infection-related readmissions (organ or space SSI and wound disruption) were the most common reasons for readmission among the shunt procedures with the highest readmission rates. These results indicate that measures to prevent SSI and wound disruption could help to reduce unplanned readmission after pediatric neurosurgery.

Readmission occurred, on average, approximately 2 weeks postoperatively. Given that postoperative infections (systemic or SSI) were the strongest predictors of read- 


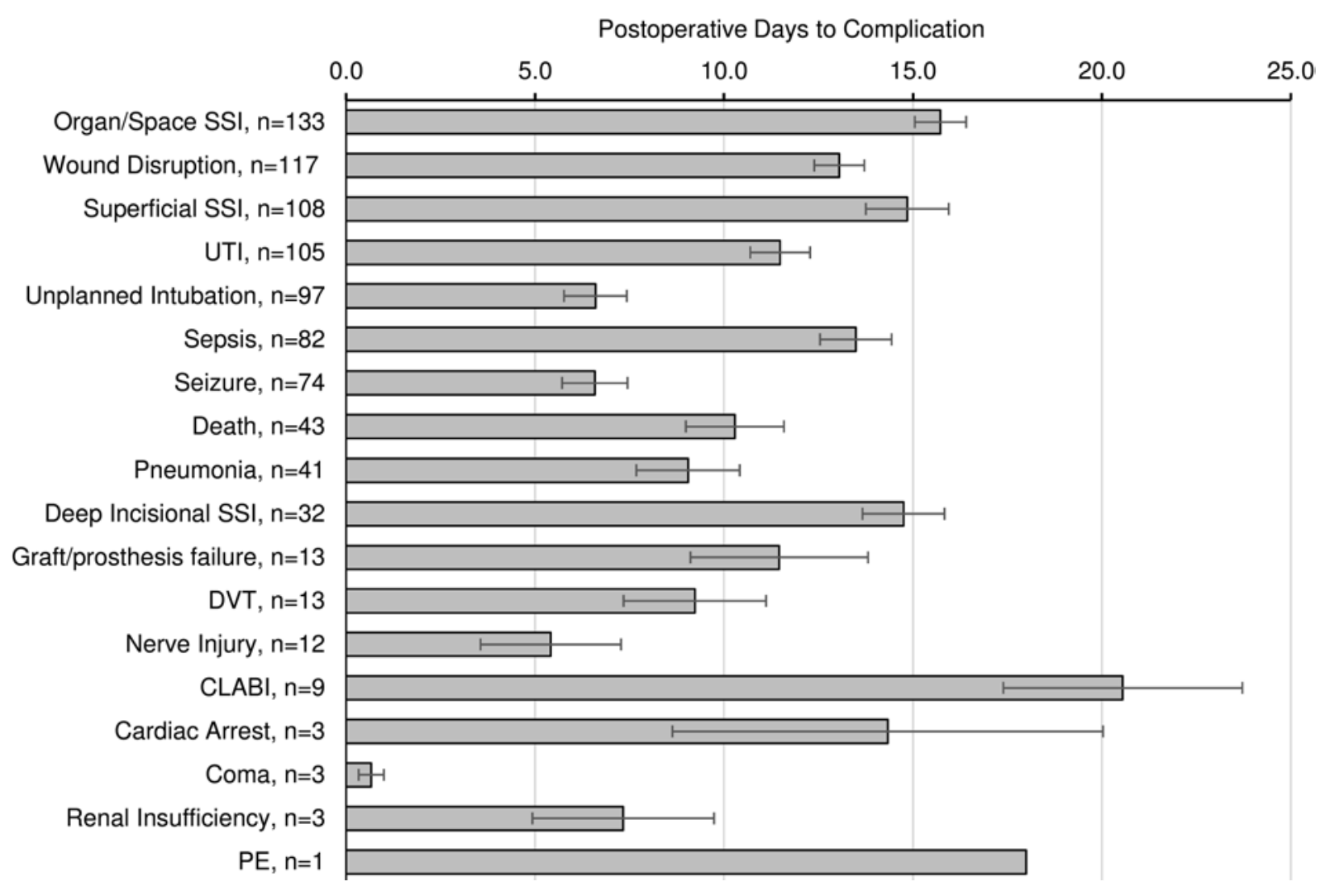

FIG. 2. Postoperative days to complication data (expressed as the mean \pm standard error of the mean with $n=$ number of events with data on days to complication). Average time to unplanned readmission was $14.04 \pm 7.74$ days postoperatively (mean \pm standard deviation). $\mathrm{CLABI}=$ central line-associated bloodstream infection.

mission, it is expected that the time course to readmission would follow a course similar to the time required for common infection complications to develop. Again, this finding highlights the need for improved postoperative wound monitoring and better operative wound management to prevent unplanned readmission. The present study supports the notion that ongoing efforts to minimize SSIs in pediatric neurosurgery have the greatest potential to reduce readmission rates.

\section{Patient-Related Factors}

The percentage of patients with any comorbidity was $91.3 \%$, and $45.3 \%$ of patients had any non-CNS comorbidity. The high comorbidity rate in pediatric neurosurgery relative to that in other specialties could be one possible cause of the higher readmission, morbidity, and mortality rates observed in this specialty relative to the rates in others. ${ }^{4}$ Comorbidities independently associated with readmission included the presence of any comorbidity, steroid use, oxygen support, nutritional support, and seizure disorder. Chronic steroid use has not been well studied in pediatric populations, although some institutions have initiated steroid avoidance protocols before renal transplantation surgery.$^{15}$ Nutritional support has been indicated as a risk factor for readmission after pediatric cardiac surgery ${ }^{16}$ and cleft palate repair. ${ }^{19}$ The comorbid conditions that have a strong association with unplanned readmission could be used as preoperative risk stratification variables. Patients who are considered high risk based on these findings may benefit from more careful discharge planning or increased outpatient attention in an effort to prevent readmission.

Native American race emerged as an independently significant risk factor for readmission. Native American race has been reported as a risk factor for readmission after orthopedic surgery ${ }^{12}$ and following sepsis, ${ }^{8}$ although its association with readmission after pediatric neurosurgical procedures has not been observed to our knowledge. While a plausible explanation for this is not apparent from these data, it is important to note disparities such as this as an area for future study. An important caveat to this observation, however, is that Native American patients make up a very small proportion of the NSQIP sample. Therefore, observations about this population are potentially subject to error given the magnified effect of small numbers of readmissions in a population with a small denominator. Furthermore, we were unable to determine socioeconomic or geographical data from the NSQIP-P data set, both of which may contribute to demographic differences in readmission rates.

\section{Procedure- and Hospital-Related Factors}

We observed a higher rate of readmission among procedures related to CSF shunts, MMC repair, and craniotomy for infratentorial tumor excision. These findings are perhaps unsurprising as these procedures are typically performed in patients with multiple comorbidities and 
TABLE 7. Postoperative complications associated with unplanned readmission on univariate analysis*

\begin{tabular}{|c|c|c|c|c|c|}
\hline \multirow[b]{2}{*}{ Complication } & \multicolumn{2}{|c|}{ №. $(\%)$} & \multirow{2}{*}{$\begin{array}{c}\text { Crude } \\
\text { OR† }\end{array}$} & \multirow[b]{2}{*}{$95 \% \mathrm{Cl}$} & \multirow[b]{2}{*}{ p Value } \\
\hline & Unplanned Readmission & No Unplanned Readmission & & & \\
\hline Any complication $\ddagger$ & $312(28.4)$ & $404(4.6)$ & 3.356 & $2.895-3.890$ & $<0.001$ \\
\hline Any infection & $219(19.9)$ & $205(2.4)$ & 10.326 & $8.431-12.646$ & $<0.001$ \\
\hline Superficial SSI & $52(4.7)$ & $56(0.6)$ & 7.674 & $5.233-11.255$ & $<0.001$ \\
\hline Deep SSI & $23(2.1)$ & $9(0.1)$ & 20.833 & $9.524-45.455$ & $<0.001$ \\
\hline Organ/space SSI & $96(8.7)$ & $37(0.4)$ & 22.435 & $15.268-32.966$ & $<0.001$ \\
\hline Sepsis & $52(4.7)$ & $30(0.3)$ & 14.369 & $9.126-22.623$ & $<0.001$ \\
\hline UTI & $35(3.2)$ & $70(0.8)$ & 4.060 & $2.692-6.122$ & $<0.001$ \\
\hline Pneumonia & $15(1.4)$ & $26(0.3)$ & 4.621 & $2.440-8.752$ & $<0.001$ \\
\hline CLABI & $3(0.3)$ & $6(0.1)$ & 3.698 & $0.991-15.873$ & 0.070 \\
\hline Wound dehiscence & $74(6.7)$ & $43(0.5)$ & 14.551 & $9.937-21.306$ & $<0.001$ \\
\hline Unplanned intubation & $20(1.8)$ & $77(0.9)$ & 2.078 & $1.265-3.412$ & 0.006 \\
\hline Renal insufficiency & $0(0.0)$ & $3(0.0)$ & NA & NA & NA \\
\hline Acute renal failure & $0(0.0)$ & $0(0.0)$ & NA & NA & NA \\
\hline Coma $>24 \mathrm{hrs}$ & $0(0.0)$ & $3(0.0)$ & NA & NA & NA \\
\hline CVA/intracranial hemorrhage & $11(1.0)$ & $31(0.4)$ & 2.830 & $1.419-5.647$ & 0.005 \\
\hline Seizure & $23(2.1)$ & $51(0.6)$ & 3.629 & $2.209-5.961$ & $<0.001$ \\
\hline Peripheral nerve injury & $1(0.1)$ & $11(0.1)$ & 1.389 & $0.179-10.766$ & 1.000 \\
\hline Cardiac arrest & $1(0.1)$ & $5(0.0)$ & 1.585 & $0.185-13.514$ & 0.510 \\
\hline Graft or prosthesis failure & $9(0.8)$ & $4(0.0)$ & 17.857 & $5.525-58.824$ & $<0.001$ \\
\hline DVT & $5(0.4)$ & $8(0.1)$ & 4.971 & $1.623-15.221$ & 0.010 \\
\hline PE & $1(0.1)$ & $0(0.0)$ & NA & NA & NA \\
\hline \multicolumn{6}{|c|}{$\begin{array}{l}\text { CLABI = central line-associated bloodstream infection. } \\
\text { * Statistical analysis was performed via binary logistic regression and Fisher's exact test. } \\
\text { † Unadjusted odds ratio with respect to each variable's null state, that is, cases in which the given complication was not present. } \\
\text { † Does not include variables not coded within NSQIP (for example, shunt failure) or perioperative or postoperative blood transfusion require- } \\
\text { ment. }\end{array}$} \\
\hline
\end{tabular}

often require close follow-up for revisions. Previous studies have shown high rates of return to system in pediatric shunt surgery, with readmission rates similar to those reported here..$^{20}$ Interestingly, 4 of the 5 procedures with the highest readmission rates accounted for over $40 \%$ of all procedures in the NSQIP-P neurosurgery cohort. Of the procedures with the highest readmission rates, operation for MMC repair was the least frequently performed (1.3\% of all procedures), and its high readmission rate is expected given the myriad comorbidities that often occur in patients with MMC. 5

Emergent or urgent triage status and admission through the emergency department were significant predictors of unplanned readmission on univariate analysis, although emergent or urgent triage was not a predictor on multivariate analysis. These findings are not surprising, yet they may aid in patient risk stratification when ascertaining patient readmission risk preoperatively.

Interestingly, a longer hospital stay was a significant protective factor in multivariate regression. This observation lends credence to the idea that for health care providers the goals of decreased hospital stay and decreased readmission may be competing. ${ }^{14}$ Efforts to decrease hospital length of stay may lead to increased readmission and vice versa. However, our data capture readmission within 30 days of the primary procedure, not from discharge; therefore, it follows that a longer hospitalization decreases the chance of readmission within 30 days because of a smaller window of time in which readmission (as defined within 30 days of the primary procedure) can occur.

\section{Study Limitations}

This study has several limitations. First, the NSQIP-P database is limited by the type of data provided by the participating institutions and by the NSQIP categorical variables. Although the NSQIP-P is a national database, the case sample is not necessarily nationally representative. Trauma cases are not included in the database. Certain procedures (for example, CPT code 62201: endoscopic third ventriculostomy with choroid plexus cauterization) are not included either. The severity of categorically coded conditions cannot be ascertained, limiting our ability to associate certain patient conditions with readmission outcomes. Some comorbidities (for example, cardiac risk factors) are insufficiently granular and do not allow for differentiation between different comorbidity subtypes (for example, atrial septal defect vs hypertrophic cardiomyopathy). Facility identifiers are not included in the NSQIP, which prevents analysis of facility outliers with substantially higher or lower rates of readmission. As is the case with many patient databases, preventative preoperative measures are not tracked, probably resulting in overestimation of risk fac- 
TABLE 8. Logistic regression analysis of variables independently associated with unplanned readmission*

\begin{tabular}{|c|c|c|c|}
\hline Variable & Adjusted OR & $95 \% \mathrm{Cl}$ & $\mathrm{p}$ Value \\
\hline Deep incisional SSI & 25.547 & $10.229-63.373$ & $<0.001$ \\
\hline Organ/space SSI & 19.156 & $11.618-31.585$ & $<0.001$ \\
\hline Wound disruption & 17.582 & $10.750-28.756$ & $<0.001$ \\
\hline Superficial incisional SSI & 12.151 & 7.783-18.973 & $<0.001$ \\
\hline Graft/prosthesis failure & 11.074 & $2.882-42.548$ & $<0.001$ \\
\hline Postop pneumonia & 4.294 & $2.045-9.017$ & $<0.001$ \\
\hline Postop UTI & 4.262 & $2.598-6.992$ & $<0.001$ \\
\hline Postop sepsis & 2.616 & $1.321-5.181$ & 0.006 \\
\hline Postop seizure & 2.532 & $1.398-4.587$ & 0.002 \\
\hline Native American race $†$ & 2.363 & $1.149-4.861$ & 0.019 \\
\hline Shunt/ventricular catheter revision, removal, or irrigation procedure & 2.283 & $1.679-3.103$ & $<0.001$ \\
\hline Shunt/ventricular catheter placement procedure $†$ & 2.128 & $1.542-2.937$ & $<0.001$ \\
\hline MMC procedure $†$ & 1.979 & $1.066-3.675$ & 0.031 \\
\hline Presence of any comorbidity & 1.943 & $1.086-3.478$ & 0.025 \\
\hline Home discharge $†$ & 1.885 & $1.208-2.942$ & 0.005 \\
\hline Oxygen supplementation $†$ & 1.645 & $1.128-2.399$ & 0.010 \\
\hline Steroid use $>10$ days & 1.411 & $1.087-1.831$ & 0.010 \\
\hline Nutritional support (IV or NG tube) & 1.403 & $1.088-1.809$ & 0.009 \\
\hline Prior operation w/in 30 days of index procedure $\dagger$ & 1.378 & $1.001-1.897$ & 0.049 \\
\hline Transfer from ER & 1.273 & $1.046-1.549$ & 0.016 \\
\hline Preexisting seizure disorder & 1.250 & $1.034-1.510$ & 0.021 \\
\hline Operation time (per hr increase) $†$ & 1.059 & $1.006-1.114$ & 0.029 \\
\hline Spine procedure & 0.703 & $0.503-0.984$ & 0.040 \\
\hline Craniotomy for craniosynostosis & 0.291 & $0.151-0.560$ & $<0.001$ \\
\hline
\end{tabular}

tors. Furthermore, not all patients had their preoperative laboratory values measured; albumin and AST had missing value rates of greater than $80 \%$, limiting our ability to interpret statistical analysis of the differences in preoperative laboratory values between groups. Data on the exact reason (that is, the ICD-9 diagnosis) for readmission in the NSQIP are not always reported or entirely clear. Of the 1098 unplanned readmissions, 746 (67.9\%) were directly related to the primary procedure. Of the remaining $32.1 \%$ of readmissions unrelated to the primary procedure, the NSQIP data abstractors may not have captured the reason for readmission, which could limit the thoroughness of our readmission reason results. Using readmission as an outcome measure has particular caveats as higher or lower rates of readmission alone may not necessarily indicate poorer or better surgical care quality.

Finally, the large number of variables analyzed increases the risk of Type I error, or false-positive findings. The Bonferroni method of multiple measures correction is very conservative, greatly decreasing the risk of incorrectly rejecting the null hypothesis (Type I error), but at the cost of increasing the risk of incorrectly accepting the null hypothesis (Type II error). In most multivariate logistic regression analyses, a higher $\alpha$ is accepted when selecting variables to include in the model in an effort to avoid inappropriately excluding important variables. Thus, we present the corrected model for reference but base our discussion on the uncorrected model.

Despite these limitations, this study may aid surgeons in identifying procedures and patient risk factors that predispose patients to a higher risk of readmission after pediatric neurosurgery. Patients undergoing longer procedures or procedures related to CSF shunting, MMC repair, or craniectomy for infratentorial brain tumor excision are at greater risk for readmission, especially if they are transferred from the emergency department. Patients undergoing spine procedures or craniotomy for craniosynostosis have a lower risk of readmission compared with those undergoing other procedures. Patients who are Native American, have any preexisting comorbidity, have undergone an operation in the previous 30 days, or have a seizure disorder should be considered at greater risk for unplanned readmission. In addition, patients who require oxygen supplementation, nutritional support, or long-term steroids should also be considered to have a greater risk for readmission. Finally, patients who experience postoperative infection (SSI or systemic infection) should be considered at the greatest risk for readmission. These data may also prove useful for family and patient counseling prior to neurosurgical operations. 


\section{Conclusions}

This is the first study to use the pediatric NSQIP data to examine hospital readmission after shunt and nonshunt neurosurgical procedures in pediatric patients. Hospital readmission rates in this study are similar to previously published rates from other sources. Unsurprisingly, SSIs and wound-related complications are some of the most important contributors to hospital readmission; therefore, efforts directed at reducing infection may have the greatest impact on readmission.

There is significant readmission rate variability among different procedure categories. Procedures with the highest rates of unplanned readmission were CSF shunt revision or removal, MMC repair, CSF shunt placement, and craniectomy for infratentorial neoplasm. Procedures with the lowest unplanned readmission rates were spine procedures, craniosynostosis craniotomies, and skin lesion procedures.

We have identified many patient-related factors associated with readmission, such as long-term steroid use, the need for nutritional support, and oxygen dependency. While these are not modifiable risk factors, they can be useful in identifying patients at high risk for readmission who could benefit from discharge planning or direct efforts to facilitate safe hospital discharge without readmission.

\section{Acknowledgments}

The American College of Surgeons National Surgical Quality Improvement Program and the hospitals participating in the ACS NSQIP are the source of the data used herein; they have not verified and are not responsible for the statistical validity of the data analysis or the conclusions derived by the authors.

\section{References}

1. American College of Surgeons: User Guide for the $\mathbf{2 0 1 3}$ ACS NSQIP Pediatric Participant Use Data File. Chicago: American College of Surgeons, 2014. (https://www.facs. org/ /media/files/quality\%20programs/nsqip/peds_puf_ userguide_2013.ashx) [Accessed February 23, 2016]

2. Axon RN, Williams MV: Hospital readmission as an accountability measure. JAMA 305:504-505, 2011

3. Berry JG, Hall DE, Kuo DZ, Cohen E, Agrawal R, Feudtner C, et al: Hospital utilization and characteristics of patients experiencing recurrent readmissions within children's hospitals. JAMA 305:682-690, 2011

4. Bruny JL, Hall BL, Barnhart DC, Billmire DF, Dias MS, Dillon PW, et al: American College of Surgeons National Surgical Quality Improvement Program Pediatric: a beta phase report. J Pediatr Surg 48:74-80, 2013

5. Bulbul A, Can E, Bulbul LG, Cömert S, Nuhoglu A: Clinical characteristics of neonatal meningomyelocele cases and effect of operation time on mortality and morbidity. Pediatr Neurosurg 46:199-204, 2010

6. Caldarelli M, Di Rocco C, La Marca F: Shunt complications in the first postoperative year in children with meningomyelocele. Childs Nerv Syst 12:748-754, 1996

7. Centers for Medicare and Medicaid Services: Medicare program; revisions to payment policies under the physician fee schedule, clinical laboratory fee schedule, access to identifiable data for the Center for Medicare and Medicaid Innovation models \& other revisions to Part B for CY 2015. Fed Regist 79:67547-68092, 2014
8. Chang DW, Tseng CH, Shapiro MF: Rehospitalizations following sepsis: common and costly. Crit Care Med 43:2085-2093, 2015

9. Chern JJ, Bookland M, Tejedor-Sojo J, Riley J, Shoja MM, Tubbs RS, et al: Return to system within 30 days of discharge following pediatric shunt surgery. J Neurosurg Pediatr 13:525-531, 2014

10. Cochrane DD, Kestle JR: The influence of surgical operative experience on the duration of first ventriculoperitoneal shunt function and infection. Pediatr Neurosurg 38:295-301, 2003

11. Cohen ME, Liu Y, Ko CY, Hall BL: Improved surgical outcomes for ACS NSQIP hospitals over time: evaluation of hospital cohorts with up to 8 years of participation. Ann Surg 263:267-273, 2009

12. Dailey EA, Cizik A, Kasten J, Chapman JR, Lee MJ: Risk factors for readmission of orthopaedic surgical patients. J Bone Joint Surg Am 95:1012-1019, 2013

13. Feudtner C, Levin JE, Srivastava R, Goodman DM, Slonim $\mathrm{AD}$, Sharma V, et al: How well can hospital readmission be predicted in a cohort of hospitalized children? A retrospective, multicenter study. Pediatrics 123:286-293, 2009

14. Jha AK, Orav EJ, Epstein AM: Public reporting of discharge planning and rates of readmissions. $\mathbf{N}$ Engl J Med 361:2637-2645, 2009

15. Lightner A, Concepcion W, Grimm P: Steroid avoidance in renal transplantation. Curr Opin Organ Transplant 16:477-482, 2011

16. Mackie AS, Gauvreau K, Newburger JW, Mayer JE, Erickson LC: Risk factors for readmission after neonatal cardiac surgery. Ann Thorac Surg 78:1972-1978, 2004

17. McLeod L, Flynn J, Erickson M, Miller N, Keren R, Dormans J: Variation in 60-day readmission for surgical-site infections (SSIs) and reoperation following spinal fusion operations for neuromuscular scoliosis. J Pediatr Orthop [epub ahead of print], 2015

18. Merkow RP, Ju MH, Chung JW, Hall BL, Cohen ME, Williams MV, et al: Underlying reasons associated with hospital readmission following surgery in the United States. JAMA 313:483-495, 2015

19. Paine KM, Paliga JT, Tahiri Y, Fischer JP, Wes AM, Wink JD, et al: An assessment of 30-Day complications in primary cleft palate repair: a review of the 2012 ACS NSQIP Pediatric. Cleft Palate Craniofac J 134 (4 Suppl 1):9, 2014

20. Piatt JH Jr: Thirty-day outcomes of cerebrospinal fluid shunt surgery: data from the National Surgical Quality Improvement Program-Pediatrics. J Neurosurg Pediatr 14:179-183, 2014

21. Sarda S, Bookland M, Chu J, Shoja MM, Miller MP, Reisner SB, et al: Return to system within 30 days of discharge following pediatric non-shunt surgery. J Neurosurg Pediatr 14:654-661, 2014

22. Sellers MM, Merkow RP, Halverson A, Hinami K, Kelz RR, Bentrem DJ, et al: Validation of new readmission data in the American College of Surgeons National Surgical Quality Improvement Program. J Am Coll Surg 216:420-427, 2013

23. Tahiri Y, Fischer JP, Wink JD, Paine KM, Paliga JT, Bartlett SP, et al: Analysis of risk factors associated with 30-day readmissions following pediatric plastic surgery: a review of 5376 procedures. Plast Reconstr Surg 135:521-529, 2015

24. University of Alabama at Birmingham Institutional Review Board: Frequently asked questions: Is IRB review required for use of public datasets? UAB Research. (http://www.uab. edu/research/administration/offices/IRB/FAQs/Pages/default. aspx?Topic=Datasets) [Accessed February 23, 2016]

25. Weiss A, Anderson JE, Chang DC: Comparing the National Surgical Quality Improvement Program with the Nationwide Inpatient Sample Database. JAMA Surg 150:815-816, 2015 
26. Wrubel DM, Riemenschneider KJ, Braender C, Miller BA, Hirsh DA, Reisner A, et al: Return to system within 30 days of pediatric neurosurgery. J Neurosurg Pediatr 13:216-221, 2014

\section{Disclosures}

The authors report no conflict of interest concerning the materials or methods used in this study or the findings specified in this paper.

\section{Author Contributions}

Conception and design: all authors. Acquisition of data: Sherrod. Analysis and interpretation of data: all authors. Drafting the article: all authors. Critically revising the article: all authors.

Reviewed submitted version of manuscript: all authors. Approved the final version of the manuscript on behalf of all authors:

Rocque. Statistical analysis: Rocque, Sherrod. Study supervision: Rocque.

\section{Supplemental Information}

Online-Only Content

Supplemental material is available with the online version of the article.

Supplement S1. http://thejns.org/doi/suppl/10.3171/2016.2. PEDS15604.

\section{Correspondence}

Brandon G. Rocque, Department of Neurological Surgery, The University of Alabama at Birmingham, 1600 7th Ave. S, Lowder 400, Birmingham, AL 35233. email: brandon.rocque@ childrensal.org. 